\title{
3 Research Square \\ miR-4319 Reduces Tumorigenicity of Cervical Cancer Cells by Targeting TUFT1
}

\section{Lijun Zheng}

Second Affiliated Hospital of Soochow University

\section{Qiongzhen Ren}

Second Affiliated Hospital of Soochow University

\section{Weipei Zhu}

Second Affiliated Hospital of Soochow University

Xiaomin Tao

Second Affiliated Hospital of Soochow University

Liangsheng Guo ( $\nabla$ gls2135@sina.com )

Second Affiliated Hospital of Soochow University

\section{Research}

Keywords: Cervical cancer, MicroRNA-4319, Proliferation, Metastasis, Tuftelin 1

Posted Date: July 7th, 2021

DOI: https://doi.org/10.21203/rs.3.rs-556240/v1

License: (c) (1) This work is licensed under a Creative Commons Attribution 4.0 International License. Read Full License 


\section{Abstract}

Background: In the present study, a new tumor suppressor function of miR-4319 was disclosed in CC.

Methods: Up-regulation of miR-4319 suppressed cell viability, proliferation, migration, invasion, and induced cell apoptosis in CC cells were measured by cell transfection, CCK-8, colony formation, EdU, flow cytometer, wound healing, transwell migration and invasion and western blot assays. Moreover, Tuftelin 1 (TUFT1) was verified as a direct target of miR-4319 by binding its 3'-UTR, confirmed by dual-luciferase reporter assay.

Result: The expression of miR-4319 was obviously decreased in clinical CC tissues and CC cell lines.

TUFT1 was remarkably increased in clinical CC tissues and CC cell lines, and was negatively associated with miR-4319 expression. Furthermore, over-expression of TUFT1 partially restored the effects of miR4319 mimic on cell viability, proliferation, migration, invasion, and cell apoptosis in CC cells.

Conclusion: miR-4319 played an anti-cancer role in the occurrence and development of CC, which might be achieved by targeting TUFT1.

\section{Introduction}

Cervical cancer (CC) is one of the most common malignant tumors in women, which occurs in the cervix and vagina (1). According to worldwide statistics, there are about 500, 000 new cases of CC every year, accounting for $5 \%$ of all new cancer cases, of which more than $80 \%$ are in developing countries $(2,3)$. Despite the increasing awareness of cancer prevention and treatment in recent years, and the popularity of related vaccines, the incidence rate of $\mathrm{CC}$ is still rising, and the age of audience becomes younger and younger (4). A large number of studies have shown that persistent high-risk human papilloma virus (HPV) infection is a relatively clear risk factor for CC. In women's life, cervical HPV infection is quite common, genital tract infection rate of HPV is more than $75 \%$, but most of them can subside by themselves $(5,6)$. It is worth emphasizing that the early symptoms of CC are not obvious, some patients are in advanced stage at the time of diagnosis, and lose the best opportunity for treatment, which seriously threatens the health and quality of women's life (7). Therefore, it is necessary to explore the potential mechanisms of $\mathrm{CC}$ for early diagnosis and effective treatment.

MicroRNAs (miRNAs) are a kind of non-coding single stranded RNA molecule with about 18-25 nucleotides in length (8). MiRNAs cause degradation or translation inhibition of target gene mRNA through specifically pairing with 3'-UTR region of target gene mRNA, further regulating the expression of target genes at post transcriptional level (9). According to statistics, miRNAs regulate at least $30 \%$ of human genes and play an important role in the normal development of the body (10). However, the disorder of miRNA expression is also common in many malignant tumors, including CC. For example, miR-21 and miR-155 are significantly over-expressed in CC tissues, and may be helpful in the prediction of both HPV positive and HPV negative cases of CC (11). MiR-501 is up-regulated, whereas CYLD protein 
is down-regulated in CC tissues, and down-regulation of miR-501 inhibits proliferation, migration and invasion, and promotes apoptosis of CC cells via targeting CYLD mediated with NF-KB p65 signaling pathway (12). MiR-1284 is down-expressed in CC tissues and CC cell lines. Up-regulation of miR-1284 suppresses proliferation and invasion, promotes apoptosis of CC cells, and enhances sensitivity of CC cells to cisplatin achieved by targeting HMGB1 (13). These findings suggest that miRNAs, acted as oncogenes or tumor suppressor genes, exhibit essential functions in the occurrence and development of CC.

MiR-4319, a newly identified cancer-related miRNA, is aberrantly expressed and becomes a predictor of patient survival in cancers (14). For instance, miR-4319 is low-expressed in colorectal cancer tissues, and over-expression of miR-4319 markedly reduces proliferation, and induces cell cycle distribution of colorectal cancer cells via targeting PLZF (15). Up-regulation of miR-4319inhibits triple-negative breast cancer (TNBC) initiation and metastasis in vivo, and suppresses the self-renewal in TNBC cancer stem cells (CSCs) through targeting E2F2 (16). Moreover, miR-4319 expression is lower in esophageal squamous cell carcinoma (ESCC) cells, and over-expression of miR-4319 represses cell growth and induces cell cycle arrest via regulating NLRC5 (17). However, the possible effects and underlying mechanisms of miR-4319 on CC remained unknown. Therefore, our intention was designed to elucidate the biological impacts and associated mechanisms of miR-4319 on CC cells.

\section{Materials And Methods 2.1 Tissue Samples}

The clinical CC tissues and adjacent tissues were obtained from $30 \mathrm{CC}$ patients who underwent surgery at the Second Affiliated Hospital of Soochow University. No radiotherapy or chemotherapy was performed before the surgery. All fresh tissues surgically resected were immediately stored at $-80^{\circ} \mathrm{C}$. The signed written informed consents were obtained from all the participants before the study.

\subsection{Cell culture and transfection}

The human CC cell lines (CaSki, SiHa, HeLa and C33A) and the cervical epithelial cells (H8) were provided by the American Type Culture Collection (ATCC, Manassas, VA, USA). Cells were cultured in Dulbecco's Modified Eagle's Medium (DMEM, Gibco, Rockville, MD, USA) containing 10\% of fetal bovine serum (FBS, Invitrogen, Grand Island, NY) and 1\% penicillin-streptomycin (Sigma- Aldrich, St. Louis, MO) in a humidified condition with $5 \% \mathrm{CO}_{2}$ at $37^{\circ} \mathrm{C}$.

GFP-miR-4319 mimic and miRNA mimic-negative control were purchased from Ambion (Austin, TX, USA). HeLa and C33A cells were cultured into 6-well plates and transfected with Lipofectamine 2000 Reagent (Invitrogen, USA) according to the manufacturer's protocols.

\subsection{Cell counting Kit-8 (CCK-8) assay}


Following the protocol of CCK-8 assay (Dojindo Laboratories, Kumamoto, Japan), the cell growth ability of transfected HeLa and C33A cells in 96-well plates was assessed at 0, 24, 48, and $72 \mathrm{~h}$, respectively. Spectrophotometer (Thermo Fisher Scientific, Waltham, MA, USA) was utilized to measure the absorbance at $450 \mathrm{~nm}$.

\subsection{Colony formation assay}

Transfected HeLa and C33A cells $\left(1 \times 10^{3} /\right.$ well) were placed in a 6-well plate, and the medium was replaced with fresh culture medium every $2-3$ days for 10 days. The colonies were fixed in $10 \%$ formaldehyde for $30 \mathrm{~min}$ and stained with $0.5 \%$ crystal violet for $5 \mathrm{~min}$. The Image-Pro Plus 6.0 (Silver Springs, MD, USA) was used for data analysis.

\subsection{Ethynyl deoxyuridine (EdU) incorporation assay}

Transfected HeLa and C33A cells $\left(1 \times 10^{4} /\right.$ well) were cultured in 96-well plates and transfected for 48 h. Then HeLa and C33A cells were fixed with $4 \%$ paraformaldehyde, Triton X-100 was used to permeabilize the nuclear membrane, and HeLa and C33A cells were blocked with goat serum for $1 \mathrm{~h}$. Furthermore, HeLa and C33A cells were stained according to the manufacturer's manual.

\subsection{Cell apoptosis by flow cytometry}

The apoptosis rate of transfected HeLa and C33A cells was detected by flow cytometry. Transfected HeLa and C33A cells were collected after 48-h transfection. Washed with PBS, FITC-labeled Annexin V and PI ( $5 \mu \mathrm{L}$ each) were added to $500 \mu \mathrm{L}$ of the cell suspension, mixed and incubated at room temperature for 5-15 $\mathrm{min}$ in the dark. Then, the rate of apoptosis was detected by flow cytometry. The experiment was repeated for 3 times.

\subsection{Wound healing assay}

Transfected HeLa and C33A cells at a density with $1 \times 10^{5} / \mathrm{mLwere}$ seeded in a 6-well plate. Then the cultured cells grew to confluence $80 \%$, the wound was made on the surface of the cell with $200 \mu \mathrm{L}$ tip gun heads. After 0 and $48 \mathrm{~h}$ respectively, HeLa and C33A cells were observed with an inverted microscope with an inverted microscopy (Tokyo, Japan), and the distance between the wounds was recorded.

\subsection{Transwell migration and invasion assays}

Transwell migration and invasion assays (Corning Inc.; USA) were performed to determine the migration and invasion capacities of HeLa and C33Acells. For transwell invasion assay, Matrigel (Becton-Dickinson Pharmingen, San Diego, CA, USA), dissolved at $4^{\circ} \mathrm{C}$ overnight, was diluted with serum-free medium and added to the upper chambers to form a gel for $30 \mathrm{~min}$ at $37^{\circ} \mathrm{C}$ with $5 \% \mathrm{CO}_{2}$. Then for transwell migration and invasion assays, transfected HeLa and C33A cells $\left(1 \times 10^{6}\right.$ cells/well $)$ were seeded into upper chamber with serum-free DMEM medium, and DMEM medium containing with $20 \%$ FBS was added in the lower chamber. After 48-h incubation, the migration and invasion cells were treated with $70 \%$ ethanol, stained with $0.5 \%$ crystal violet, and counted by an inverted microscope (Tokyo, Japan). 


\subsection{RNA Extraction and qRT-PCR}

TRIzol reagent (Invitrogen, Carlsbad, CA, USA) was utilized to extract the total RNA from clinical CC tissues and cell lines. Through the reverse Transcription Kit (TaKaRa Biotechnology Co., Ltd., Dalian, China), the total RNA was reversely transcribed to complementary deoxyribose nucleic acids (cDNAs). Routine qRT-PCR was implemented using ABI 7300-fast RT PCR system (Applied Biosystems) with SYBR Green PCR Kit (Qiagen) based on the specifications. Relative expressions of miRNA and mRNA were evaluated by $2^{-\triangle \triangle C t}$ method with $U 6$ or $\beta$-actin as internal reference. The primer sequences used were listed as below: miR-4319 forward, 5'-CACCCAGAGCAAAGCCAC-3', miR-4319 reverse, 5'GTGCAGGGTCCGAGGT-3', TUFT1 forward, 5'-TGGTCTGAGCTCGCCATCAGTTTCA-3', TUFT1 reverse, 5'CCCTGAGGGACCAGCCACATAGAACAGA-3', U6 forward, 5'-CTCGCTTCG GCAGCACA-3', U6 reverse, 5'AACGCTTCACGAATTTGCGT-3', $\beta$-actin forward, 5'-AGAAGGC TGGGGCTCATTTG-3', $\beta$-actin reverse, 5 'AGGGGCCATCCACAGTCTTC-3'. Conditions for qRT-PCR were used: $95^{\circ} \mathrm{C}$ for $10 \mathrm{~min}, 40$ cycles of $95^{\circ} \mathrm{C}$ for $15 \mathrm{~s}$, and $60^{\circ} \mathrm{C}$ for $1 \mathrm{~min}$

\subsection{Western blot analysis}

The protein of CC cells was lysed in RIPA lysis buffer. After $12,000 \mathrm{~g}$ centrifugation for $15 \mathrm{~min}$ at $4^{\circ} \mathrm{C}$, the total protein concentrations were determined by BCA protein assay kit (Beyotime, Haimen, China). Equivalent samples were separated using 10\% SDS-PAGE and then transferred onto a PVDF membrane for $2 \mathrm{~h}$.Then they were blocked with 5\% nonfat skim milk in Tris-Buffered Saline and Tween-20 (TBS-T) buffer at room temperature for $1 \mathrm{~h}$ and incubated with the following primary antibodies at $4^{\circ} \mathrm{C}$ overnight: anti-Bcl-2 (1: 1, 000, ab32124), anti-Bax (1: 1, 000, ab32053), anti-cleaved caspase-3 (1: 1, 000, ab32042), anti-cleaved caspase-9 (1: 1, 000, ab2324), anti-Cox-2 (1: 1, 000, ab15191), anti-MMP-2 (1: 1, 000, ab97779), anti-MMP-9 (1: 1, 000, ab38898), anti-TUFT1 (1: 1, 000, ab184949), anti- $\beta$-actin (1: 1, 000, ab8226). After the primary antibodies were washed three times with TBS-T, the second antibody was added and incubated for $2 \mathrm{~h}$ at room temperature. Enhanced Chemiluminescence Detection System was carried out to evaluate the protein expressions. $\beta$-actin was used as the loading control. All antibodies were purchased from Abcam (Cambridge, MA, USA).

\subsection{Dual-luciferase reporter assay}

3'-untranslated region (3'-UTR) of TUFT1 was cloned into the pGL3 vector (Promega, Madison, WI, USA), which was identified as wild-type (WT) 3'-UTR. The quick-change site-directed mutagenesis kit (Stratagene, Cedar Creek, USA) was used for site-directed mutagenesis of the miR-4319 binding site in TUFT1 3'-UTR, which was named as mutant (MUT) 3'-UTR. Cells were co-transfected with WT-3'UTR/MUT-3'-UTR and NC mimic or miR-4319 mimic for $48 \mathrm{~h}$. Dual-luciferase reporter assay system (Promega, Madison, WI, USA) was used for determining luciferase activity.

\subsection{Statistical analysis}


Statistical analysis was conducted by the Statistical Product and Service Solutions (SPSS) 17.0 (SPSS Inc., Chicago, IL, USA). The Student's t-test was performed to compare inter group differences. Data were presented as mean \pm standard deviation (SD). $P<0.05$ was considered of statistical significance.

\section{Results}

\section{1 miR-4319 expression is down-regulated in clinical tissues and cancer cell lines}

To explore the possible effects and underlying mechanisms of miR-4319on CC, firstly, qRT-PCR assay was performed to evaluate the expression of miR-4319 in clinical CC tissues and cell lines. The data of Fig. 1A showed that miR-4319 expression was significantly down-regulated in CC tissues compared with that in normal tissues. Besides, as expected, the expression of miR-4319 was also decreased in CC cells (especially in HeLa and C33A cells) relative to that in $\mathrm{H} 8$ cells (Fig. 1B). These data suggested that miR4319 was down-regulated in CC tissues and cell lines, and functioned as a tumor suppressor gene in the occurrence and development of CC. To further investigate the functional role of miR-4319 in CC cells, miR-4319 mimic and NC mimic were transfected into HeLa and C33A cells, and transfection efficiency was assessed. As shown in Fig. 1C and 1D, the expression of miR-4319 was significantly increased in HeLa and C33A cells transfected with miR-4319 mimic compared with NC mimic group.

\subsection{Up-regulation of miR-4319 inhibits viability, proliferation, and induces apoptosis of CC cells}

Firstly, CCK-8 assay was adopted to evaluate the effects of miR-4319 on the viability of HeLa and C33A cells, and the data of Fig. 2A displayed that miR-4319 mimic significantly inhibited the viability of HeLa and C33A cells in a time-dependent manner compared with NC mimic group. Besides, colony formation and EdU assays were carried out to assess the effects of miR-4319 on the proliferation of HeLa and C33A cells, and the data of Fig. $2 \mathrm{~B}$ and $2 \mathrm{C}$ showed that up-regulation of miR-4319 obviously suppressed the proliferation of HeLa and C33A cells compared with NC mimic group. Moreover, the effects of miR-4319 on the apoptosis of HeLa and C33A cells was determined by flow cytometry, and the data of Fig. 2D indicated that compared with NC mimic group, over-expression of miR-4319 remarkably promoted the apoptosis of HeLa and C33 cells. Furthermore, western blot was performed to evaluate the effects of miR4319 on the expression levels of apoptosis-related proteins including Bax, Bcl-2, Cleaved-caspase-3 and Cleaved-caspase-9 in HeLa and C33A cells. The data of Fig. 2E showed that relative to NC mimic group, miR-4319 mimic notably reduced the protein expression of Bcl-2, but promoted the protein levels of Bcl-2, Cleaved-caspase- 3 and Cleaved-caspase- 9 both in HeLa and C33A cells. These data suggested that upregulation of miR-4319 inhibited viability, proliferation, and induced apoptosis of CC cells.

\subsection{Up-regulation of miR-4319 inhibits migration and invasion of $\mathrm{CC}$ cells}


Firstly, wound healing assay was performed to further explore the functional effects of miR-4319 on the migration of CC cells. As shown in Fig. 3A, compared with the NC mimic group, miR-4319 mimic obviously inhibited the migration of HeLa and C33A cells. Then transwell migration and invasion assays were also carried out, and the data of Fig. 3B suggested that compared with NC mimic group, overexpression of miR-4319 significantly inhibited the migration and invasion of HeLa and C33A cells. Furthermore, western blot was performed to evaluate the effects of miR-4319 on the expression levels of metastasis-related proteins, including Cox-2, MMP-2 and MMP-9. The data of Fig. $3 \mathrm{C}$ revealed that relative to NC mimic group, miR-4319 mimic notably decreased the protein expressions of Cox-2, MMP-2 and MMP-9 in HeLa and C33A cells. These data suggested that up-regulation of miR-4319 inhibited migration and invasion of CC cells.

\subsection{TUFT1 is direct target gene of miR-4319, and negatively associated with miR-4319}

In order to study on the possible target genes of miR-4319 involved in the occurrence of CC,firstly, TargetScan was carried out to predict that TUFT1 was potential candidate of miR-4319 (Fig. 4A). TUFT1, as an important regulator, has been reported to control the occurrence and development of various tumors $(18,19)$. In addition, dual-luciferase reporter gene analysis was performed to verify whether miR4319 directly regulated TUFT1, and the results of Fig. 4B indicated that co-expression with miR-4319 mimic significantly inhibited the activity of a firefly luciferase reporter containing wild-type TUFT1 3'-UTR, while this did not be detected on a reporter with a mutated TUFT1 3'-UTR. The data suggested that TUFT1 was a direct target of miR-4319. To further confirm the targeting relationship between miR-4319 and TUFT1, qRT-PCR and western blot assays were used to determine the mRNA and protein expressions of TUFT1 in HeLa and C33A cells transfected with miR-4319 mimic. The data of Fig. 4C and 4D showed that compared with NC mimic group, the mRNA and protein expression levels of TUFT1 were obviously downregulated in HeLa and C33A cells transfected with miR-4319 mimic. Moreover, qRT-PCR assay was carried out to assess the TUFT1 in clinical CC tissues and CC cell lines, and the data of Fig. 4E and 4F illustrated that TUFT1 was obviously up-regulated in clinical CC cancer tissues and CC cell lines. These data suggested that TUFT1 was direct target gene of miR-4319, and was negatively associated with miR-4319 in CC.

\subsection{Up-regulation of miR-4319 inhibits viability, proliferation, migration and invasion, and induced apoptosis of CC cells via targeting TUFT1}

Pc-TUFT1 was transfected into HeLa and C33A cells, and qRT-PCR was performed so as to explore whether miR-4319 functioned as a tumor suppressor gene through targeting TUFT1. The data of Fig. 5A showed that after transfection with pc-TUFT1, the expression of TUFT1 was obviously increased in HeLa and C33A cells. Then, CCK-8 was performed to evaluate the effects of TUFT1 on the viability of HeLa and C33A cells. As shown in Fig. 5B, up-regulation of TUFT1 obviously restored the inhibitory effects of miR4319 mimic on the viability of HeLa and C33A cells. Besides, EdU assay showed that over-expression of TUFT1 obviously retarded the inhibitory effects of miR-4319 mimic on the proliferation of HeLa and C33A 
cells (Fig. 5C). In addition, the results of Fig. 5D indicated that up-regulation of TUFT1 obviously inhibited promoting effects of miR-4319 mimic on the apoptosis of HeLa and C33A cells. Furthermore, transwell migration and invasion results displayed that the up-regulation of TUFT1 obviously antagonized the inhibitory effects of miR-4319 mimic on the migration and invasion of HeLa and C33A cells. These data suggested that up-regulation of miR-4319 inhibited viability, proliferation, migration and invasion, and induced apoptosis of CC cells via targeting TUFT1.

\section{Discussion}

The etiology of CC involves complex molecular mechanisms, including activation of oncogenes and inhibition of tumor suppressor genes (20). With the continuous research on the molecular mechanisms of CC, more and more genes and proteins involved in the occurrence of CC have been reported, which promotes the diversity of treatment methods for $\mathrm{CC}$, including gene targeted therapy, hormone therapy and so on $(21-23)$. In view of this, to explore the molecular mechanisms of the occurrence, metastasis and recurrence of $\mathrm{CC}$, to find markers for clinical diagnosis and prognosis of $\mathrm{CC}$, and to identify effective molecular targets have become the research hotspot of medical personnel in various countries. In view of the extensive existence of miRNAs and the diversity of gene regulation, much more attention has been paid to the role of miRNAs in the development and tumorigenesis in recent years (24-26). Among them, miR-4319 has been confirmed as a regulatory factor of gene mRNA transcription, regulating the post transcriptional expression of specific target genes, and then participating in the pathological process of many cancers (27-29). However, the expression of miR-4319 in human CC and its biological mechanisms remained unclear.

It has been confirmed that miR-4319 is involved in the pathological process of many diseases, and is recognized as a tumor suppressor gene, which can be used as an independent factor of clinical prognosis of many cancers. For example, miR-4319 acts as a tumor suppressor in non-small cell lung cancer (NSCLC) progression via restraining cell proliferation and migration as well as boosting apoptosis through inhibiting LIN28-mediated RFX5 stability (30). MiR-4319 is notably down-regulated in thyroid cancer tissues and cells.Up-regulation of miR-4319 attenuates proliferation, migration, and epithelial-tomesenchymal transition (EMT) in thyroid cancer via targeting SMURF1 (31). Moreover, miR-4319 level is decreased in prostate cancer (PC) specimens, and re-expression of miR-4319 in PC cells inhibits Her-2dependent cell growth (32). Similarly, our study displayed that miR-4319 was low-expressed in CC tissues and cell lines. Up-regulation of miR-4319 repressed viability, proliferation, migration, invasion, and induced apoptosis of CC cells, suggesting that miR-4319 was an inhibitory factor of CC, which might participate in the occurrence and development of CC.

MiRNAs produce a marked effect through inducing mRNA degradation or translation inhibition by base pairing with 3 '-UTR of target genes $(33,34)$. At present, post transcriptional regulation mechanisms of miRNAs on target genes play an important role in tumor development including CC (35-37). In this study, TUGT1 was highly expressed in CC tissues and cell lines. TUGT1 was predicted as a candidate target of miR-4319 by TargetScan, which was further confirmed by dual-luciferase reporter assay. TUFT1 gene is 
identified, cloned and sequenced from ameloblast cDNA library by Deutsch et al $(38,39)$. TUFT1 is located at 1dq21.3, which is involved in the growth and maturation of extracellular enamel (39). At present, studies have focused on its relationship with enamel mineralization and tooth development. However, TUFT1 exists in many tissues, such as kidney, adrenal gland, liver and testis (40). Furthermore, recent studies have shown that TUFT1 is abnormally expressed in many kinds of tumors, and is closely related to the development of multiple tumors. TUFT1 is over-expressed in pancreatic cancer (PC), and depletion or over-expression of TUFT1 correspondingly inhibits or promotes PC cell migration and metastasis in vitro and in vivo, through regulation of EMT (18). TUFT1 is elevated in hepatocellular carcinoma (HCC), and promotes HCC cell growth, metastasis and EMT in vitro and in vivo via activation of $\mathrm{Ca}^{2+} / \mathrm{PI} 3 \mathrm{~K} / \mathrm{AKT}$ pathway (19). TUFT1 expression is increased in breast cancer samples. Inhibition of TUFT1 expression in T-47D and MDA-MB-231 breast cancer cells significantly affects cell proliferation, induced apoptosis, and leads to G1-phase cell cycle arrest (39). These findings were closely in line with our studies.Through rescue experiment, over-expression of TUFT1 partially restored the effects of miR4319 mimic on viability, proliferation, migration and invasion of CC cells.

In conclusion, the expression ofmiR-4319 was decreased, while the expression of TUFT1 was increased in clinical CC tissues and cell lines. Up-regulation of miR-4319 inhibited the growth and metastasis of CC cells via targeting TUFT1, which provided research basis for gene targeted therapy of CC in the future.

\section{Declarations}

\section{Ethic approval}

The experimental protocol was established, according to the ethical guidelines of the Helsinki Declaration and was approved by the Animal Ethics Committee of Affiliated Huai'an Hospital of Xuzhou Medical University

\section{Consent for publication}

This manuscript has no consent for publication

\section{Availability of data and materials}

Data sharing is not applicable to this article as no datasets were generated or analysed during the current study.

\section{Competing interests}

All authors have no conflicts of interest

\section{Funding}

No funding was received during this study 


\section{Author's contributions}

Lijun Zheng and Liangsheng Guo designed the study; Lijun Zheng, Qiongzhen Ren, Weipei Zhu, Xiaomin Tao did the experiment; Lijun Zheng, Qiongzhen Ren, Weipei Zhu, Xiaomin Tao acquired and analysed the data; Liujun Zheng drafted the manuscript and Liangsheng Guo review the manuscript.

\section{Acknowledgement}

Not applicable

\section{References}

1. C Zhang et al. The direct and indirect association of cervical microbiota with the risk of cervical intraepithelial neoplasia. Cancer Med. 2018;7:2172.

2. NC Nkfusai et al. Cervical cancer in the Bamenda Regional Hospital, North West Region of Cameroon: a retrospective study. Pan Afr Med J. 2019;32:90.

3. M Saeed et al. A Synopsis on the Role of Human Papilloma Virus Infection in Cervical Cancer. Curr Drug Metab. 2018;19:798.

4. M Wei et al. Rising Mortality Rate of Cervical Cancer in Younger Women in Urban China. J Gen Intern Med. 2019;34:281.

5. Hu Z, Ma D. The precision prevention and therapy of HPV-related cervical cancer: new concepts and clinical implications. Cancer Med. 2018;7:5217.

6. Rizzo AE, Feldman S. Update on primary HPV screening for cervical cancer prevention. Curr Probl Cancer. 2018;42:507.

7. Dueñas-González A, Campbell S. Global strategies for the treatment of early-stage and advanced cervical cancer. Curr Opin Obstet Gynecol. 2016;28:11.

8. Hayes J, Peruzzi PP, Lawler S. MicroRNAs in cancer: biomarkers, functions and therapy. Trends Mol Med. 2014;20:460.

9. Van Roosbroeck K, Calin GA. Cancer Hallmarks and MicroRNAs: The Therapeutic Connection. Adv Cancer Res. 2017;135:119.

10. Mansoori B, Mohammadi A, Shirjang S, Baradaran B. MicroRNAs in the Diagnosis and Treatment of Cancer. Immunol Invest. 2017;46:880.

11. Park S, et al. MiR-9, miR-21, and miR-155 as potential biomarkers for HPV positive and negative cervical cancer. BMC Cancer. 2017;17:017.

12. JGP Sanches et al. miR-501 is upregulated in cervical cancer and promotes cell proliferation, migration and invasion by targeting CYLD. Chem Biol Interact. 2018;285:85.

13. Chen J, Li G. MiR-1284 enhances sensitivity of cervical cancer cells to cisplatin via downregulating HMGB1. Biomed Pharmacother. 2018;107:997. 
14. M Yang et al. Long noncoding RNA ITGB2-AS1 promotes growth and metastasis through miR4319/RAF1 axis in pancreatic ductal adenocarcinoma. J Cell Physiol. 2020;20:29471.

15. Huang L, et al. MiR-4319 suppresses colorectal cancer progression by targeting ABTB1. United European Gastroenterol J. 2019;7:517.

16. Chu J, et al. MiR-4319 Suppress the Malignancy of Triple-Negative Breast Cancer by Regulating SelfRenewal and Tumorigenesis of Stem Cells. Cell Physiol Biochem. 2018;48:593.

17. Hu X, et al. miR-4319 Suppresses the Growth of Esophageal Squamous Cell Carcinoma Via Targeting NLRC5. Curr Mol Pharmacol. 2020;13:144.

18. B Zhou et al. TUFT1 regulates metastasis of pancreatic cancer through HIF1-Snail pathway induced epithelial-mesenchymal transition. Cancer Lett. 2016;382:11.

19. Dou C, et al. Hypoxia-induced TUFT1 promotes the growth and metastasis of hepatocellular carcinoma by activating the $\mathrm{Ca}(2+) / \mathrm{PI} 3 \mathrm{~K} / \mathrm{AKT}$ pathway. Oncogene. 2019;38:1239.

20. P Tsikouras et al. Cervical cancer: screening, diagnosis and staging. J Buon. 2016;21:320.

21. Li H, Wu X, Cheng X. Advances in diagnosis and treatment of metastatic cervical cancer. J Gynecol Oncol. 2016;27:e43.

22. Menderes G, Black J, Schwab CL, Santin AD. Immunotherapy and targeted therapy for cervical cancer: an update. Expert Rev Anticancer Ther. 2016;16:83.

23. Brucker SY, Ulrich UA. Surgical Treatment of Early-Stage Cervical Cancer. Oncol Res Treat. 2016;39:508.

24. Laengsri V, Kerdpin U, Plabplueng C, Treeratanapiboon L, Nuchnoi P. Cervical Cancer Markers: Epigenetics and microRNAs. Lab Med. 2018;49:97.

25. B Pardini et al. MicroRNAs as markers of progression in cervical cancer: a systematic review. BMC Cancer. 2018;18:018.

26. AJ. Granados-López et al. Use of Mature miRNA Strand Selection in miRNAs Families in Cervical Cancer Development. Int J Mol Sci. 2017;18:407.

27. Han S, et al. MiR-4319 induced an inhibition of epithelial-mesenchymal transition and prevented cancer stemness of HCC through targeting FOXQ1. Int J Biol Sci. 2019;15:2936.

28. F Albano et al. SETBP1 and miR_4319 dysregulation in primary myelofibrosis progression to acute myeloid leukemia. J Hematol Oncol. 2012;5:48.

29. Wang Y, Zhao H, Zhi W. SEMA4D under the posttranscriptional regulation of HuR and miR-4319 boosts cancer progression in esophageal squamous cell carcinoma. Cancer Biol Ther. 2020;21:122.

30. Yang Y, et al. MiR-4319 hinders YAP expression to restrain non-small cell lung cancer growth through regulation of LIN28-mediated RFX5 stability. Biomed Pharmacother. 2019;115:14.

31. Bian S. miR-4319 inhibited the development of thyroid cancer by modulating FUS-stabilized SMURF1. J Cell Biochem. 2020;121:174.

32. Lin X, Wang Y. Re-expression of microRNA-4319 inhibits growth of prostate cancer via Her-2 suppression. Clin Transl Oncol. 2018;20:1400. 
33. Wu J, Zhao Y, Li F, Qiao B. MiR-144-3p: a novel tumor suppressor targeting MAPK6 in cervical cancer. J Physiol Biochem. 2019;75:143.

34. Xiao $\mathrm{H}$, et al. MiR-340 suppresses the metastasis by targeting EphA3 in cervical cancer. Cell Biol Int. 2018;42:1115.

35. Li N, Cui T, Guo W, Wang D, Mao L. MiR-155-5p accelerates the metastasis of cervical cancer cell via targeting TP53INP1. Onco Targets Ther. 2019;12:3181.

36. Q Hu et al. miR-146a promotes cervical cancer cell viability via targeting IRAK1 and TRAF6. Oncol Rep. 2018;39:3015.

37. Li Q, Yu X, Yang L. MiR-145 inhibits cervical cancer progression and metastasis by targeting WNT2B by Wnt/ $\beta$-catenin pathway. Int J Clin Exp Pathol. 2019;12:3740.

38. Y Leiser et al. The induction of tuftelin expression in PC12 cell line during hypoxia and NGF-induced differentiation. J Cell Physiol. 2011;226:165.

39. W Liu et al. TUFT1 is expressed in breast cancer and involved in cancer cell proliferation and survival. Oncotarget. 2017;8:74962.

40. D Deutsch et al. The human tuftelin gene and the expression of tuftelin in mineralizing and nonmineralizing tissues. Connect Tissue Res. 2002;43:425.

\section{Figures}

A

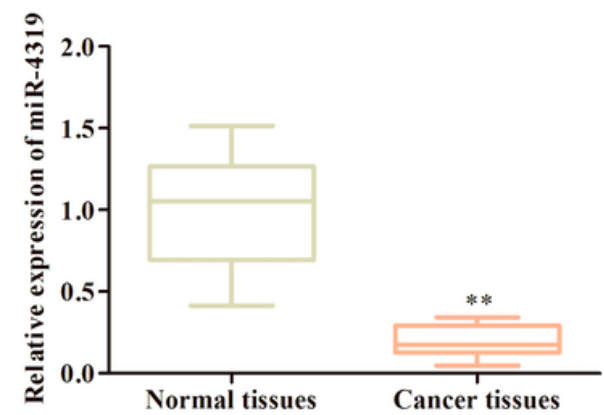

B

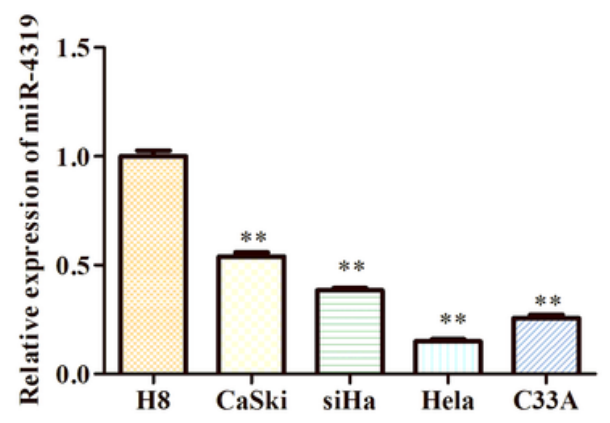

$\mathrm{D}$
C

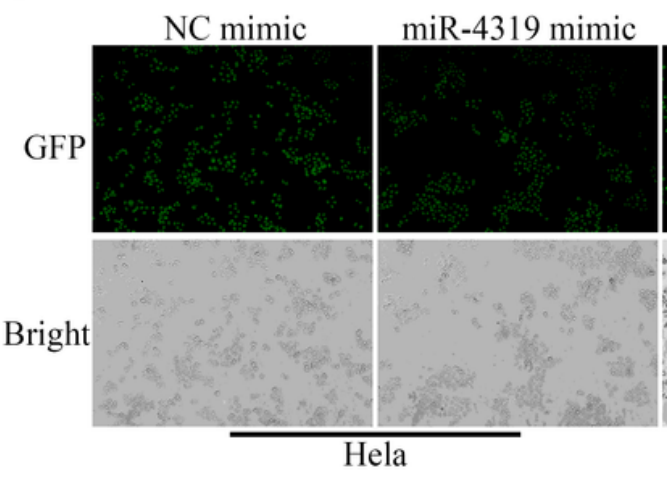

$\mathrm{NC}$ mimic

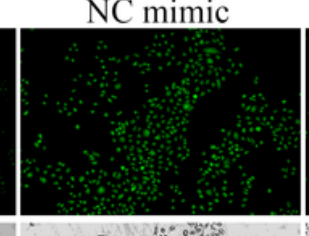

miR-4319 mimic

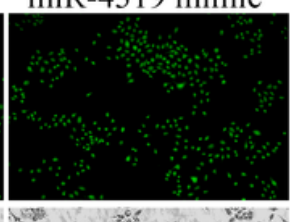

NC mimic

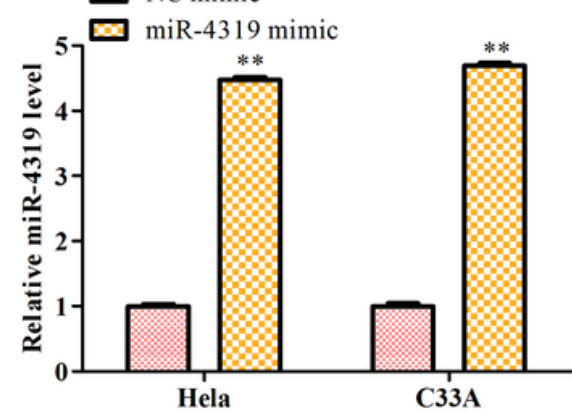

Figure 1 
miR-4319 is down-regulated in clinical tissues and cell lines. The expression of miR-4319in (A) CC tissues and (B) cell lines was detected by qRT-PCR assay. ${ }^{*} \mathrm{P}<0.01$ vs. Normal tissues or H8 cells. (C) GFP and (D) qRT-PCR assays were performed to evaluate the miR-4319 expression in HeLa and C33A cells after transfection with miR-4319 mimic. ${ }^{*} \mathrm{P}<0.05,{ }^{*} \mathrm{P}<0.01 \mathrm{vs}$. NC mimic. All data were presented as mean \pm SD. $n=3$.

A
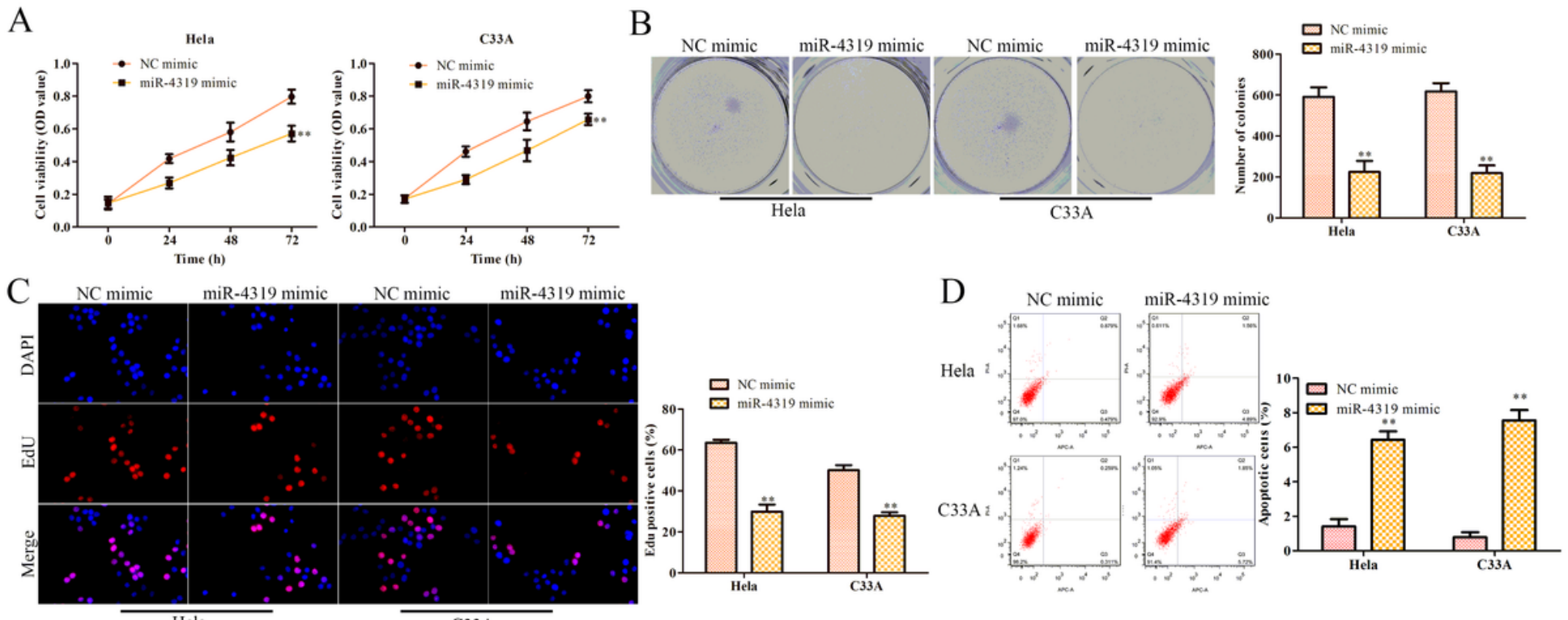

E
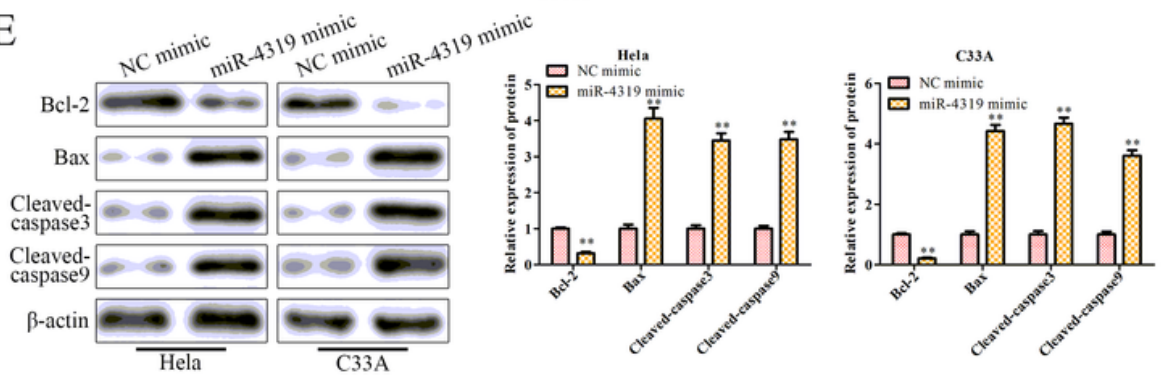

Figure 2

Up-regulation of miR-4319 inhibits viability, proliferation, and induces apoptosis of CC cells. (A) The viability of HeLa and C33A cells transfected with miR-4319 mimic was assessed by CCK-8 assay at indicated times. The proliferation of HeLa and C33A cells transfected with miR-4319 mimic was evaluated by (B) colony formation and (C) EdU assays. (D) The apoptotic rate of HeLa and C33A cells transfected with miR-4319 mimic was determined by flow cytometry assay. (E) The expression levels of proteins, including Bax, Bcl-2, Cleaved-caspase-3 and Cleaved-caspase-9, were evaluated by western blot assay. ${ }^{\star} \mathrm{P}<0.01$ vs. NC mimic. All data were presented as mean $\pm S D$. $n=3$. 

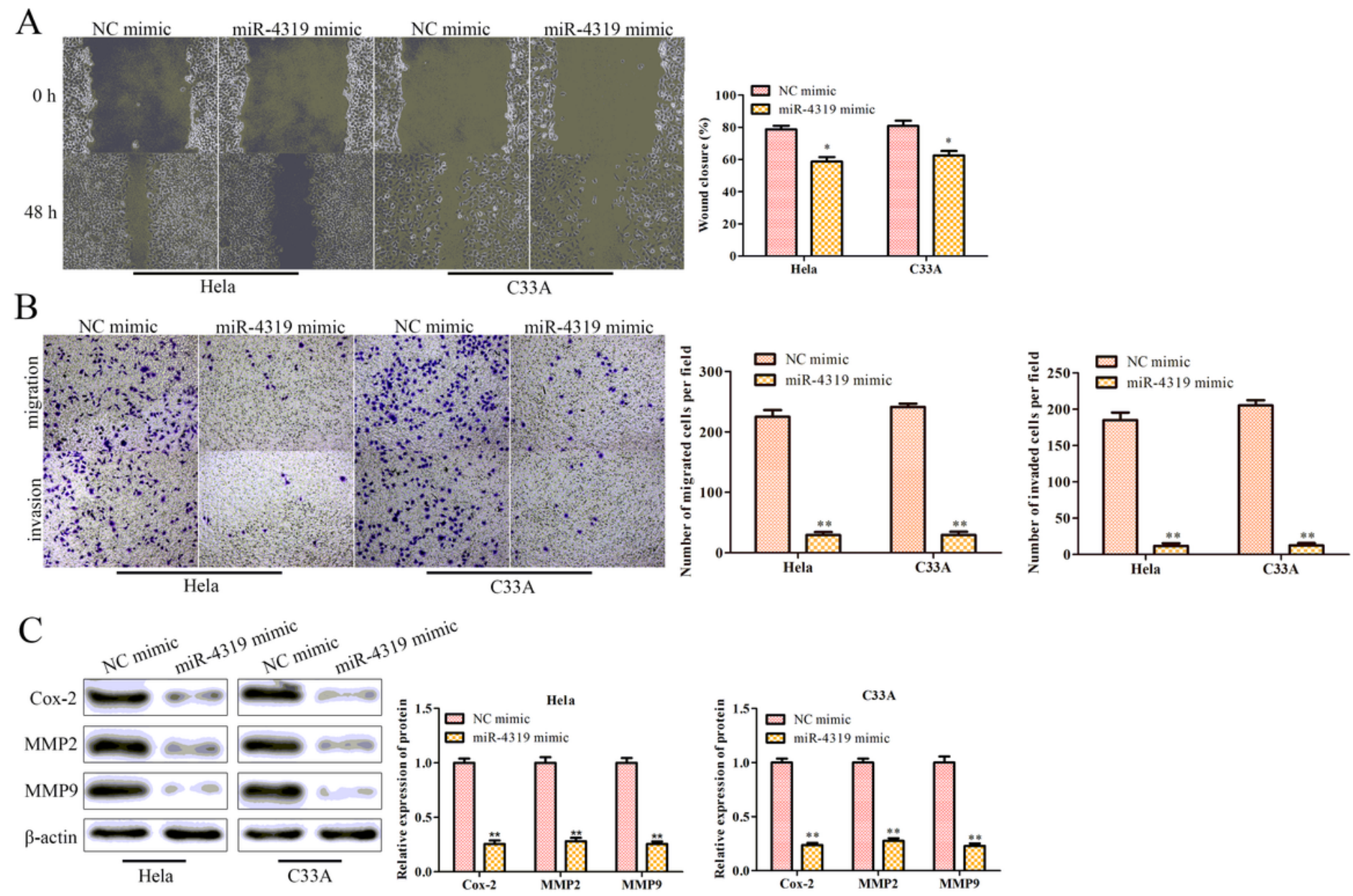

\section{Figure 3}

Up-regulation of miR-4319 inhibits migration and invasion of CC cells. (A) The migration of HeLa and C33A cells transfected with miR-4319 mimic was determined by wounding healing assay. (B) The migration and invasion of HeLa and C33A cells transfected with miR-4319mimic was determined by transwell migration and invasion assays. (C) The expression levels of proteins, including Cox-2, MMP-2 and MMP-9, were evaluated by western blot assay. ${ }^{*} P<0.05,{ }^{\star} \mathrm{P}<0.01$ vs. NC mimic. All data were presented as mean \pm SD. $\mathrm{n}=3$. 


\begin{tabular}{|c|c|}
\hline TUFT1 WT & 5 '-aauGCUCUGGGAUUUCAGGGa-3' \\
\hline & || || |||||||| \\
\hline Hsa-miR-4319 & 3 '-cacCGAAAC---------GAGUCCCu-5 ' \\
\hline TUFT1 Mut & 5 '-aauCGAAACGGAUGAGUCCCa-3' \\
\hline
\end{tabular}
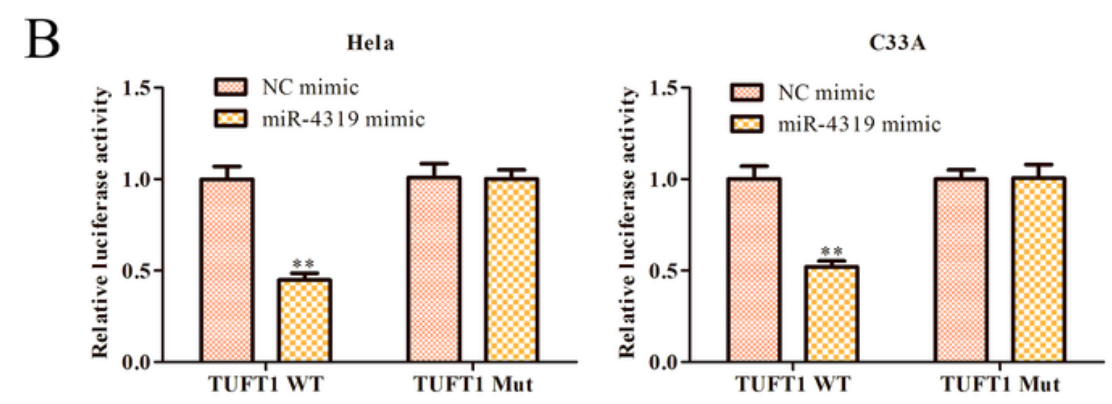

C

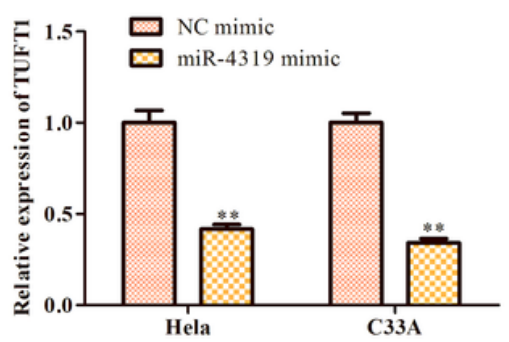

$\mathrm{D}$

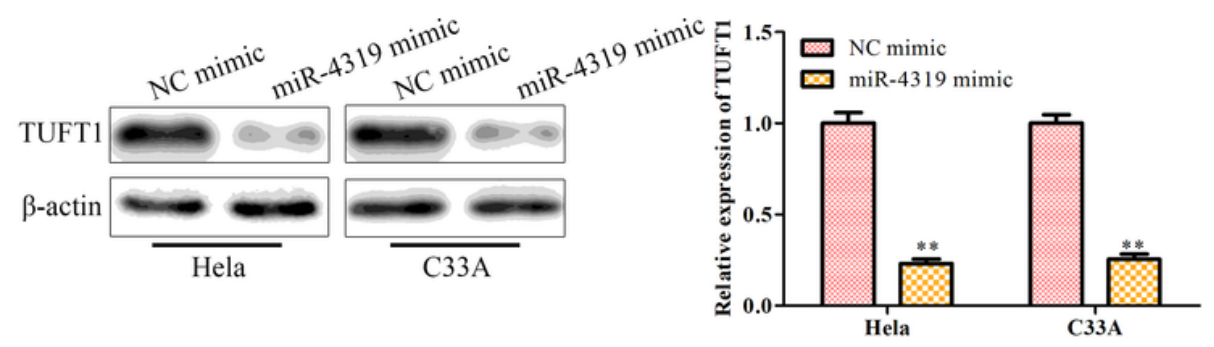

$\mathrm{E}$
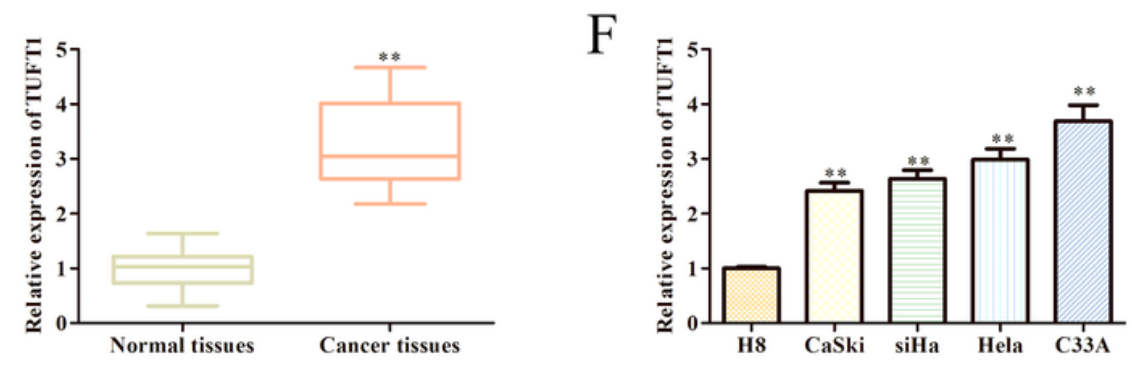

Figure 4

TUFT1 is direct target gene of miR-4319, and negatively associated with miR-4319. (A) The binding sites between miR-4319 and TUFT1. (B) Luciferase-reporter analysis was employed to validate the coactions between miR-4319 and TUFT1. (C) qRT-PCR assay was performed to evaluate the TUFT1 expression in HeLa and C33A cells transfected with miR-4319 mimic. (D) Western blot assay was performed to evaluate the TUFT1 expression in HeLa and C33A cells transfected with miR-4319 mimic. ${ }^{\star * P}<0.01$ vs. NC mimic. The expression of TUFT1 in (E) CC tissues and (F) cell lines was detected by qRT-PCR assay. ${ }^{\star *} \mathrm{P}<0.01$ vs. Normal tissues or $\mathrm{H} 8$ cells. All data were presented as mean $\pm \mathrm{SD}$. $\mathrm{n}=3$ 

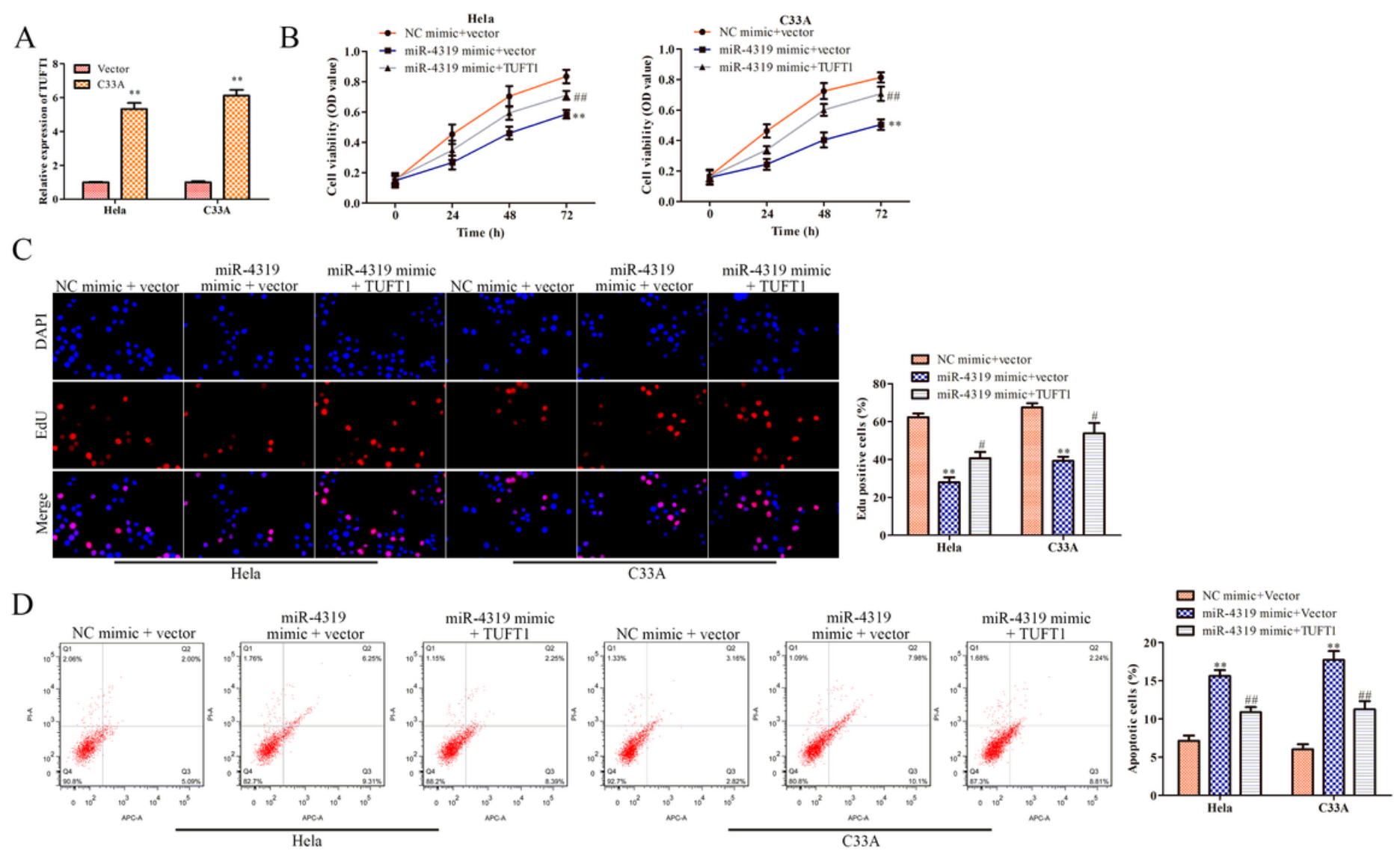

E
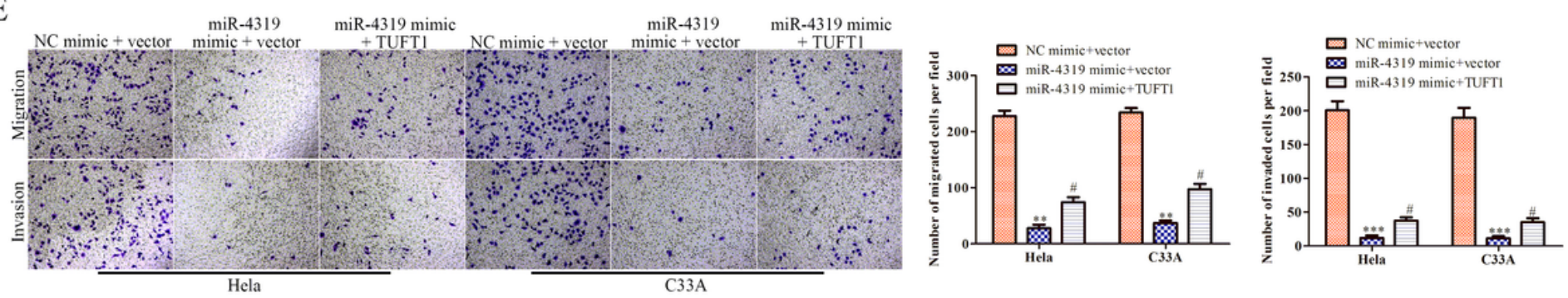

Figure 5

Up-regulation of miR-4319 inhibits viability, proliferation, migration and invasion, and induced apoptosis of CC cells via targeting TUFT1. (A) The expression of TUFT1 in HeLa and C33A cells transfected with pcTUFT1 was evaluated by qRT-PCR assay. ${ }^{\star * P}<0.01$ vs. Vector. (B) The viability of HeLa and C33A cells transfected with pc-TUFT1 was assessed by CCK-8 assay at indicated times. (C) The proliferation of HeLa and C33A cells transfected with pc-TUFT1 was evaluated by EdU assay. (D) The apoptotic rate of HeLa and C33A cells transfected with pc-TUFT1 was determined by flow cytometry assay. (E) The migration and invasion of HeLa and C33A9 cells transfected with pc-TUFT1 was determined by Transwell migration and invasion assays. ${ }^{\star *} \mathrm{P}<0.01$ vs. NC mimic + vector. \#P<0.05, \#\#P<0.01 vs. miR-4319 mimic + vector. All data were presented as mean \pm SD. $n=3$. 